PHYSICAL REVIEW E 66, 026307 (2002)

\title{
Influence of ohmic heating on the flow field in thin-layer electrodeposition
}

\author{
Matthias Schröter* and Klaus Kassner \\ Fakultät für Naturwissenschaften, Otto-von-Guericke Universität Magdeburg, Postfach 4120, D-39016 Magdeburg, Germany \\ Ingo Rehberg \\ Physikalisches Institut, Universität Bayreuth, D-95440 Bayreuth, Germany \\ Josep Claret and Francesc Sagués \\ Departament de Química-Física, Universitat de Barcelona, Martí i Franquès 1, E-08028 Barcelona, Spain
}

(Received 10 April 2002; published 21 August 2002)

\begin{abstract}
In thin-layer electrodeposition the dissipated electrical energy leads to a substantial heating of the ion solution. We measured the resulting temperature field by means of an infrared camera. The properties of the temperature field correspond closely with the development of the concentration field. In particular, we find that the thermal gradients at the electrodes act similar to a weak additional driving force to the convection rolls driven by concentration gradients.
\end{abstract}

DOI: 10.1103/PhysRevE.66.026307

PACS number(s): 47.27.Te, 87.63.Hg, 81.15.Pq

\section{INTRODUCTION}

The electrochemical deposition of metals from aqueous solutions in quasi-two-dimensional geometries has proven to be a valuable test bed to examine concepts of interfacial growth such as fractal growth [1,2], morphological transitions [3-5], or dendritic growth [6,7]. The properties of the evolving deposit are in many cases sensitive to the presence of convection currents in the solution [8-13]. Two different convection effects have been found in these experiments: (a) at small length scales, electroconvection appears between the tips of the growing deposit [14,15]. Although some approaches have been addressed to understand this convection mechanism, it still lacks a conclusive theoretical description. (b) Density inhomogeneities due to concentration changes at the electrodes induce large scale gravity-driven convection rolls $[7,10,13,15-19]$. In this case, the experimental flow field [13] is found to be in quantitatively good agreement with theoretical predictions [18].

However, there is another potential source of density changes: due to the small cell volume of typically $\leqslant 1 \mathrm{~cm}^{3}$, the dissipated electric energy can be the source of a significant heating. If the cell were completely thermally insulated, a solution exposed to an electrical power of $500 \mathrm{~mW}$ would start to boil after $500 \mathrm{~s}$. While thermal conduction will confine the overall temperature increase to smaller values, considerable temperature gradients might arise and generate density driven convection.

Thermally induced convection rolls have been thoroughly studied in thin-layer geometries heated from below (for a recent review see Ref. [20]) and the side [21-25]. In contrast, the role of thermal effects was previously not examined in electrodeposition. In order to quantify the possible temperature gradients, a high spatial resolution of the temperature field is necessary. We present here measurements of the

\footnotetext{
*Electronic address: matthias.schroeter@physik.uni-magdeburg.
}

temperature field at both electrodes performed by use of an infrared camera. The so determined evolution of the temperature field can be related to the development of the concentration field, which is known from interferometric measurements $[7,26]$. The remainder of the paper is organized as follows. Section II describes our experimental setup. Section III presents the measurement results, which will be discussed in Sec. IV.

\section{EXPERIMENTAL SETUP}

The measurements are performed with the infrared camera Varioscan 3021-ST from InfraTec, which contains a Stirling-cooled $\mathrm{HgCdTe}$ detector with $360 \times 240$ pixel. Using its macro we observe an area of $5.0 \times 3.4 \mathrm{~cm}^{2}$, which yields a spatial resolution of $140 \mu \mathrm{m}$. The maximal image capturing frequency is $1.1 \mathrm{~Hz}$, the thermal resolution $\pm 30 \mathrm{mK}$.

While the sensor is sensitive for wavelengths in the range of 8-12 $\mu \mathrm{m}$, glass plates, which are normally used as top and bottom plate of the cell, are opaque in this region. Therefore the upper cell cover was realized using a polyethylene foil stretched over an aluminum frame. Due to the flexibility of the polyethylene foil and a small overpressure necessary to fill the cell, the plate separation of about $0.5 \mathrm{~mm}$ is not well defined. The bottom plate of the cell consists of a block of Teflon, because this material has a low reflectivity in the infrared. This reduces the so called narcissism: the response of the cooled detector to its own mirror image. The electrodes are parallel zinc wires (Goodfellow 99.99\%) of 0.25 $\mathrm{mm}$ diameter and separated by a distance of $4 \mathrm{~cm}$. Figure 1 shows an image of the cell during an experiment.

The cell is filled with an $0.1 \mathrm{M} \mathrm{ZnSO}_{4}$ solution prepared from Merck p.a. chemicals in nondeaerated ultrapure $\mathrm{H}_{2} \mathrm{O}$. The measurements are performed at a constant potential of $20 \pm 0.003 \mathrm{~V}$. Due to the current increase during the electrodeposition process, the average electrical power feed $\dot{Q}_{\mathrm{el}}$ increases from $470 \mathrm{~mW}$ at the beginning of the experiment to $650 \mathrm{~mW}$ after $500 \mathrm{~s}$.

Because of the modified cell construction, the question of 


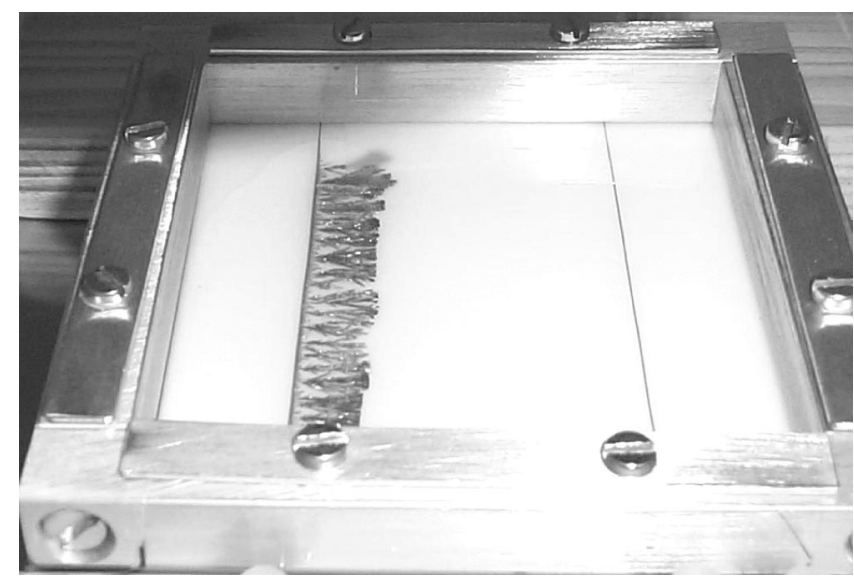

FIG. 1. Cell used for the infrared measurements. The bottom (white) consists of Teflon, the upper cover is a polyethylene foil stretched over an aluminum frame. The electrodes are parallel zinc wires with a distance of $4 \mathrm{~cm}$. The deposit at the cathode has grown for $410 \mathrm{~s}$.

comparability with experiments performed in standard electrodeposition cells could be raised. Therefore we calculate the heat flux $k_{i} A_{i}$ for the confining plates, where $A_{i}$ and $k_{i}$ are the area and the heat transfer coefficient of the plate. It is important to keep in mind, that $1 / k_{i}$ is equivalent to $1 / \alpha_{1}$ $+1 / \alpha_{2}+\Delta z_{i} / \lambda_{i}$. Here $\alpha_{1}$ and $\alpha_{2}$ are the heat transition numbers from solution to plate, respectively, plate to air, $\lambda_{i}$ is the heat conductivity, and $\Delta z_{i}$ the thickness of the plate.

Inserting the material parameters of our setup [27,28] and using only the area between the two electrodes, we derive a $k_{i} A_{i}$ of $16.2 \mathrm{~mW}$ per $\mathrm{K}$ temperature difference for the polyethylene foil and $11.5 \mathrm{~mW} / \mathrm{K}$ for the Teflon plate, while a typical glass plate (Schott BK 7, $6.3 \mathrm{~mm}$ thick) yields 16 $\mathrm{mW} / \mathrm{K}$. So in a first approximation our setup is thermally equivalent to a standard electrodeposition cell.

\section{EXPERIMENTAL RESULTS}

Figure 1 shows the growing deposit at the cathode, which belongs to the homogenous morphology [29]. It is characterized by tip-splitting but retaining a growing front parallel to the cathode. After $500 \mathrm{~s}$ a Hecker transition [4] takes place and the growth front breaks up into more spatially localized zones of active development.

Immediately before each experiment the infrared camera takes a zero image, which is then subtracted from all images taken during the experiment. Therefore the thermographies solely depict the temperature increase. Figure 2 gives an example of such a thermography after $280 \mathrm{~s}$, the scale at the right describes the temperature increase with respect to the beginning of the experiment. The white line marks the position of the anodic zinc wire. The temperature decrease at the left hand side of the image is caused by the thermal conductivity of the aluminum frame, the warm "island" in the middle is due to the inhomogeneity of the cell thickness.

In order to improve the signal to noise ratio, zones of spatial homogeneity and a width of $9.1 \mathrm{~mm}$ are chosen by visual inspection. Such a zone is depicted in Fig. 2 with two parallel black lines. Inside this zone all rows are averaged, yielding a temperature increase $\Delta T(y, t)$, which is only a function of distance to the cathode $y$ and time.

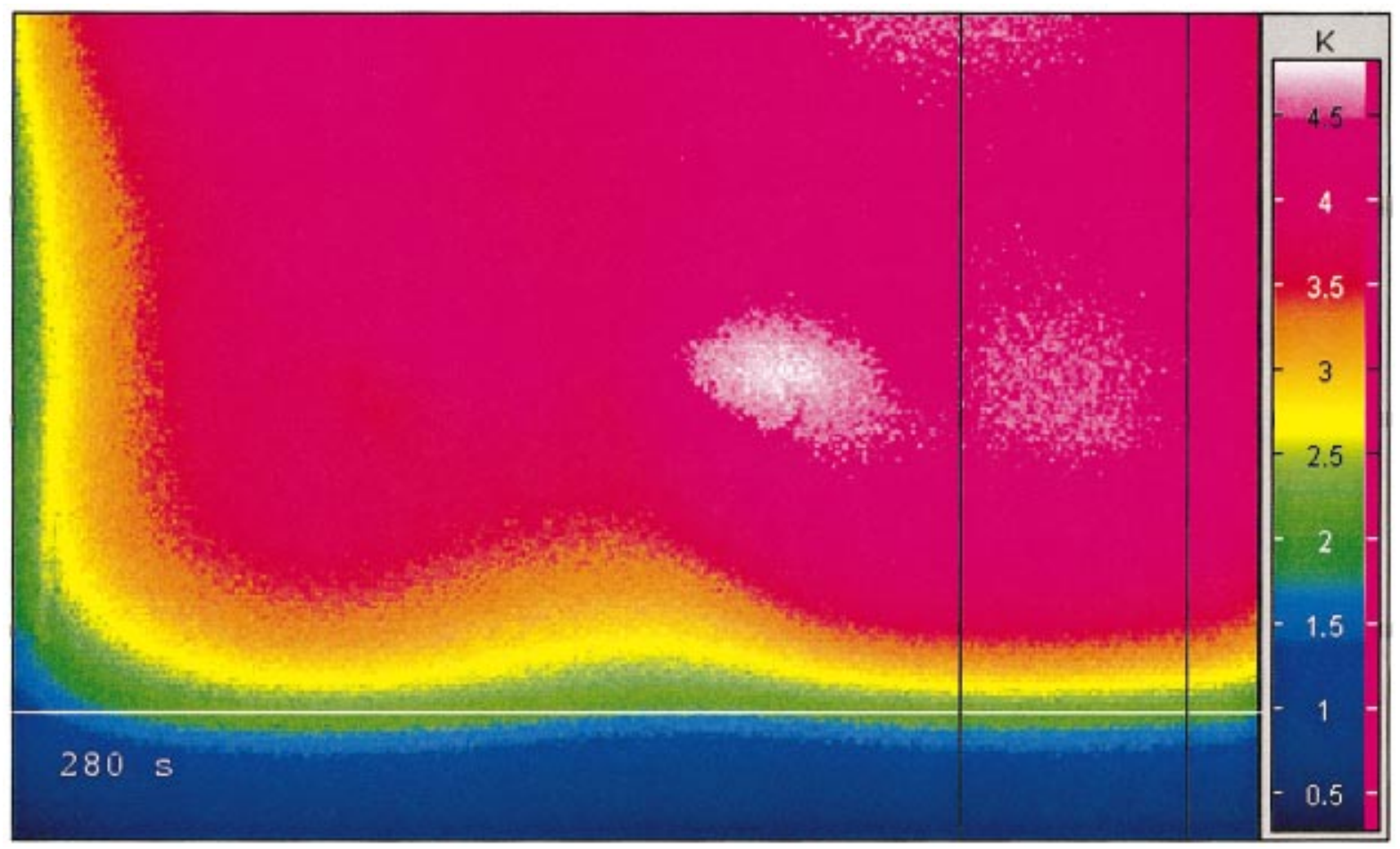

FIG. 2. (Color) Thermography taken $280 \mathrm{~s}$ after the start of the experiment. The size of the image is $5.0 \times 3.4 \mathrm{~cm}^{2}$. The white line corresponds to the position of the anodic zinc wire, the rows between the black lines are averaged to produce Fig. 3. 


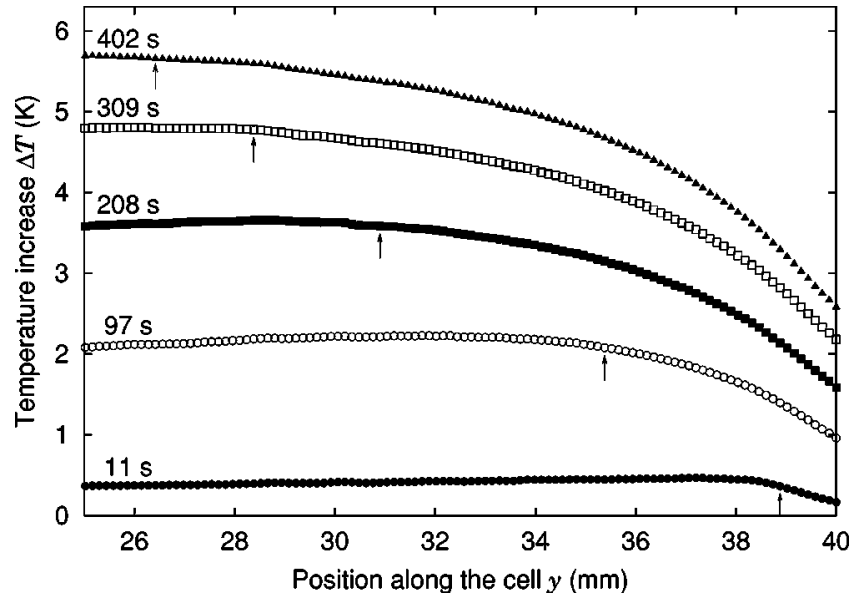

FIG. 3. Temporal evolution of the temperature field at the anode, which is located at $40 \mathrm{~mm}$. All data are averaged over a width of 9.1 $\mathrm{mm}$. The arrows indicate the position where the deviation of $\Delta T$ from the bulk temperature becomes less than $20 \mathrm{mK}$.

Figure 3 shows the evolution of $\Delta T$ in the neighborhood of the anode. It is clearly visible that the temperature increase at the anode itself lags behind with respect to the one observed in the middle of the cell.

Figure 4 illustrates the evolution of the temperature field at the cathode. The most prominent feature is the existence of a local temperature maximum, denoted with small arrows. This maximum moves towards the middle of the cell, where a plateau of spatially constant temperature is located.

In order to characterize the heating process in the cell, we pick the temperature at a distance of $25.6 \mathrm{~mm}$ to the cathode (this is approximately the location, where the two convection rolls emerging from the electrodes finally meet, as will be discussed in Sec. IV B). Referring to this point, we will speak of the bulk in the following. In Fig. 5 this temperature increase $\Delta T_{\text {bulk }}$ is given for the two experiments presented in Figs. 3 and 4 . The fact that the two measurements are almost identical reflects the reproducibility of the experiment. The

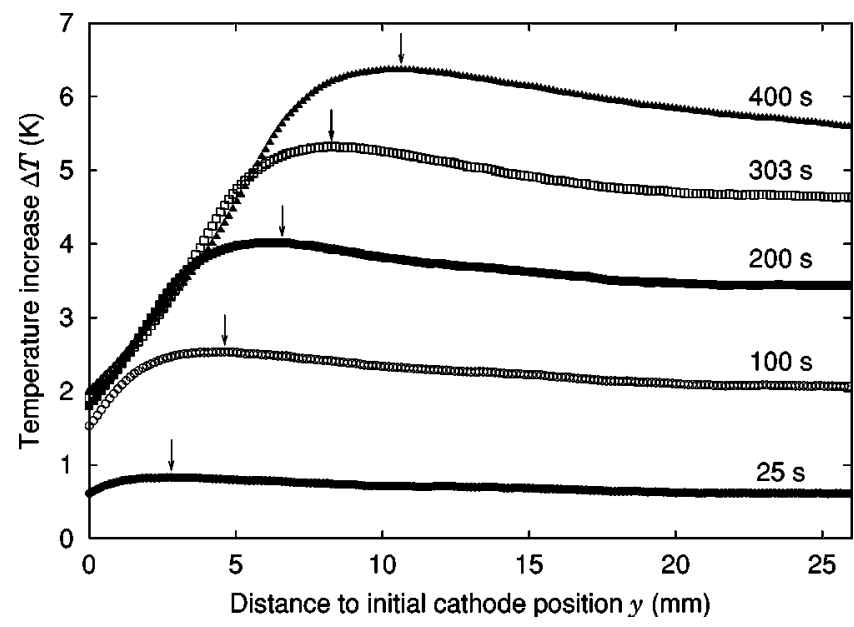

FIG. 4. Temporal evolution of the temperature field at the cathode. All data are averaged over a width of $9.1 \mathrm{~mm}$. The arrows mark the position of the temperature maximum.

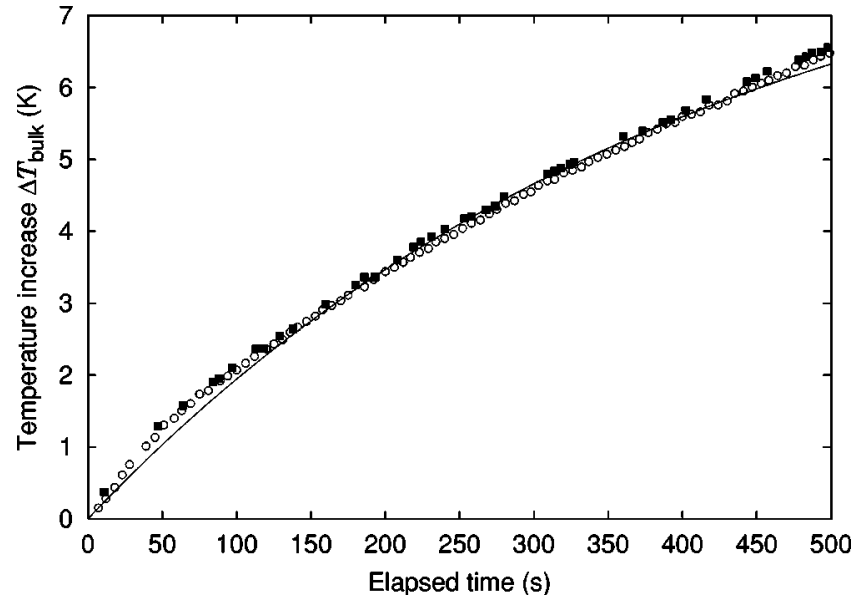

FIG. 5. Temperature increase in the bulk of the cell at a distance of $14.4 \mathrm{~mm}$ to the anode and $25.6 \mathrm{~mm}$ to the cathode. The $\bigcirc$ correspond to the experiment presented in Fig. 4 the $\mathbf{\square}$ to the one in Fig. 3. The solid line is a fit of Eq. (5) to the $\bigcirc$.

solid line is a fit to an exponential function, which is motivated in detail in Sec. IV C.

The arrows in Fig. 3 represent the location, where the temperature is $20 \mathrm{mK}$ smaller than $\Delta T_{\text {bulk }}$. The distance of these points with respect to the anode is denoted $L_{a}(t)$ and is shown in Fig. 6(a). Its monotonous increase with time is fitted by a power law, which will be explained in Sec. IV A. Correspondingly, Fig. 6(b) shows the growth of the distance $L_{c}(t)$ between the the location of the temperature maximum in Fig. 4 and the cathode. The straight line is a fit to all data with $t>100 \mathrm{~s}$, which will be motivated in Sec. IV B.

\section{DISCUSSION AND CONCLUSIONS}

In principle, the energy balance involves three contributions: the electrical energy feed into the cell, the dissipated ohmic heat and the chemical reaction energy. However, the short calculation presented in the appendix supports the assumption that the chemical energy contribution is mostly irrelevant.

The ohmic heat dissipated at some position in the cell will be proportional to the local resistivity $\rho(y, t)$ in a onedimensional model, while $\rho$ will depend on the local ion concentration $c(y, t)$. In the next three subsections we will compare the evolution of the temperature field at the anode, at the cathode, and in the bulk with the development of the concentration field, which is known from interferometric measurements $[7,26]$.

\section{A. Evolution of the temperature field at the anode}

At the anode $\mathrm{Zn}^{2+}$ ions go into solution, increasing the local concentration and therefore density. While this denser solution sinks down to the bottom plate, it gets replaced by less dense bulk solution. This mechanism drives a convection roll of size $L[13,15,18]$. According to the fluid dynamical description there are two growth regimes: Initially during the so-called immiscible fluid regime $L$ will grow with $t^{0.8}$, while after some time there will be a crossover to the diffu- 


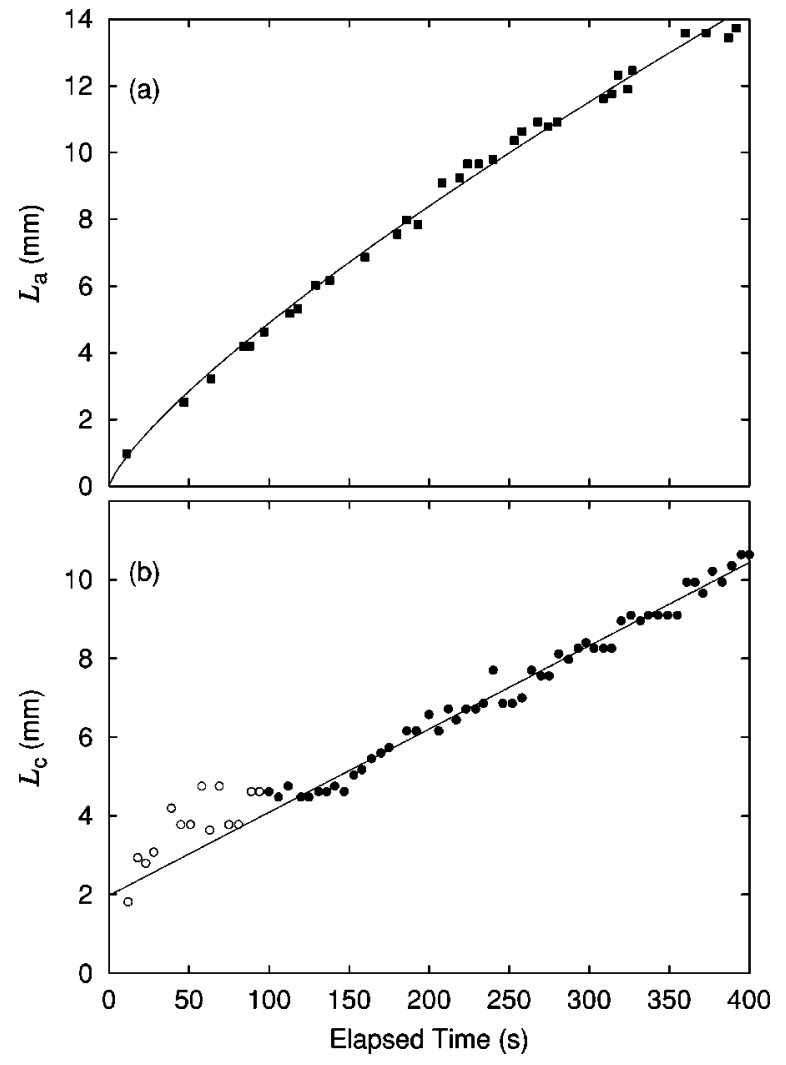

FIG. 6. Temporal evolution of (a) the distance between the anode and the point, where the temperature starts to deviate from the bulk. The solid line is a fit with Eq. (1). (b) the distance between the temperature maximum and the initial cathode position. The fit is performed with Eq. (2) to the data indicated with filled symbols.

sion hindered spreading regime with a $t^{0.5}$ growth law. Chazalviel et al. [18] showed that both the fluid velocity $v$ and the ion concentration decrease with increasing distance to the electrode. The distance, where $v$ has dropped to zero and coincidently $c$ has fallen to $c_{\text {bulk }}$, defines $L$.

Figure 3 shows that the temperature increase in the region between the anode and the arrows lags behind the one observed in the bulk. As $c>c_{\text {bulk }}$ translates into reduced ohmic heating, we identify the position of the arrows with the end of the anodic convection roll. A fit to its length with

$$
L_{\mathrm{a}}(t)=a t^{b}
$$

is presented in Fig. 6(a). It yields $a=0.13 \pm 0.01 \mathrm{~mm}$ and $b=0.78 \pm 0.01$, which indicates that the convection roll is in the immiscible fluid regime for the whole run of the experiment. According to the scaling analysis presented in Ref. [13], this corresponds to an average plate separation of about $650 \mu \mathrm{m}$

\section{B. Evolution of the temperature field at the cathode}

Due to the growing deposit, there is a zone of ion depletion in the vicinity of the cathode, the size of which will be affected by the convection roll driven by the occuring density difference. As the decreased $c$ leads to higher dissipation, the increased temperature denoted by the arrows in Fig.
4 is qualitatively explained. The distance of the arrows to the initial cathode position $L_{\mathrm{c}}(t)$ should correspond to the actual size of the deposit. Therefore we perform a linear fit with

$$
L_{\mathrm{c}}(t)=v_{\mathrm{c}} t+l_{\mathrm{c}},
$$

which is shown in Fig. 6(b). We derive $v_{\mathrm{c}}=21.2$ $\pm 0.4 \mu \mathrm{m} / \mathrm{s}$, which agrees well with $20.7 \pm 0.8 \mu \mathrm{m} / \mathrm{s}$ front velocity determined from photographs of the deposit.

$l_{\mathrm{c}}$ is found to be $2 \pm 0.1 \mathrm{~mm}$. This finite distance can be explained by the absence of heat production in the metallic deposit because of its low resistivity and the fact that its heat conductivity is 190 times higher than water. So the deposit is an effective heat sink, the resulting heat flux shifts the temperature maximum into the cell.

If the cathodic convection roll, apart from the fact that it starts at the actual front of the deposit, grows in the same way as the anodic roll, they meet $450 \mathrm{~s}$ after the beginning of the experiment at a distance of $25 \mathrm{~mm}$ to the cathode. This corresponds to the observed change in morphology after that time.

\section{Temperature evolution in the bulk}

The temperature increase observed in Fig. 5 can be modeled if we assume that the whole cell shares the constant bulk properties $c$ and $\rho$ and therefore $T$. The supplied electrical power $\dot{Q}_{\mathrm{el}}$ would then be compensated by the heating of the system with heat capacity $C$ and the heat flow $\dot{Q}_{\text {flow }}$ :

$$
\dot{Q}_{\text {flow }}=-\left(T-T_{0}\right) \sum_{i} k_{i} A_{i},
$$

where $T_{0}$ represents the ambient temperature and $T$ the temperature inside the electrolyte. If we assume $\dot{Q}_{\text {el }}$ to be constant, the corresponding differential equation

$$
\dot{Q}_{\mathrm{el}}=\left(T-T_{0}\right) \sum_{i} k_{i} A_{i}+C \frac{\partial T}{\partial t}
$$

has the straightforward solution

$$
\left(T-T_{0}\right)=T_{\text {final }}\left(1-e^{-t / \tau}\right) .
$$

Here $T_{\text {final }}=\dot{Q}_{\text {el }} / \sum_{i} k_{i} A_{i}$ denotes the finally reached temperature difference and $\tau=C / \Sigma_{i} k_{i} A_{i}$ is the time constant of the heating up. A fit of Eq. (5) to the experimental data is displayed in Fig. 5. It yields $T_{\text {final }}=9 \pm 0.2 \mathrm{~K}$. This translates to an overall heat flux $\Sigma_{i} k_{i} A_{i}$ of $60 \mathrm{~mW}$ per K temperature difference. A comparison of this result with the values of $k_{i} A_{i}$ calculated in Sec. II shows that about $50 \%$ of the heat flux takes place through the top and the bottom plate of the cell. The heat flux through the side walls, the electrodes and the plate area beyond the electrodes accounts for the rest.

\section{Influence of the temperature gradients on the convection rolls}

In order to estimate the influence of the temperature gradients on the concentration driven convection currents, we 


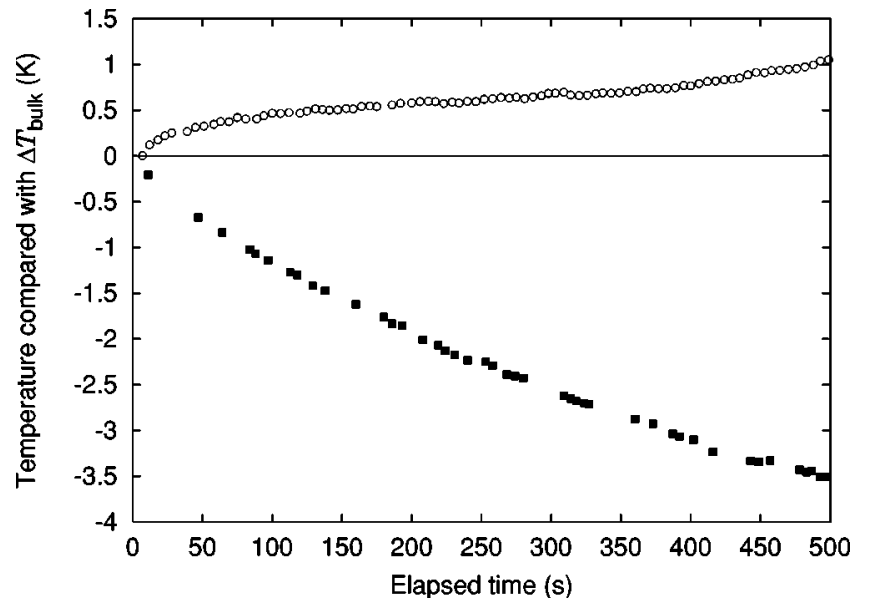

FIG. 7. Temperature difference between the bulk and $\bigcirc$ : the cathodic temperature maximum and $\mathbf{\square}$ : the temperature at the anode.

plot in Fig. 7 the difference between $\Delta T_{\text {bulk }}$ and (a) the cathodic temperature maximum and (b) the anode. These temperature gradients result in a down-flow at the anode and an up-flow at the growth front, so they act as an additional driving.

For a more quantitative determination of their contribution, the temperature dependency of the density was measured for different concentrations. The applied density measurement instrument DMA 5000 from Anton Paar has an accuracy better than $50 \mu \mathrm{g} / \mathrm{cm}^{3}$.

After $400 \mathrm{~s}$ the temperature of the $0.1 \mathrm{M} \mathrm{ZnSO}_{4}$ bulk solution has reached about $30{ }^{\circ} \mathrm{C}$ which corresponds to a density of $1012.3 \mathrm{mg} / \mathrm{cm}^{3}$. In accordance with the measurements presented in Ref. [7] and the theory in Ref. [18], we estimate $c$ at the anode for that time as $0.25 \mathrm{M}$. This corresponds to a density of $1036.4 \mathrm{mg} / \mathrm{cm}^{3}$ for $T=30^{\circ} \mathrm{C}$ and $1037.4 \mathrm{mg} / \mathrm{cm}^{3}$ for the actually measured $T \approx 27^{\circ} \mathrm{C}$. So the contribution of the temperature gradient to the overall density difference at the anode is about $4 \%$ as visualized in Fig. 8.

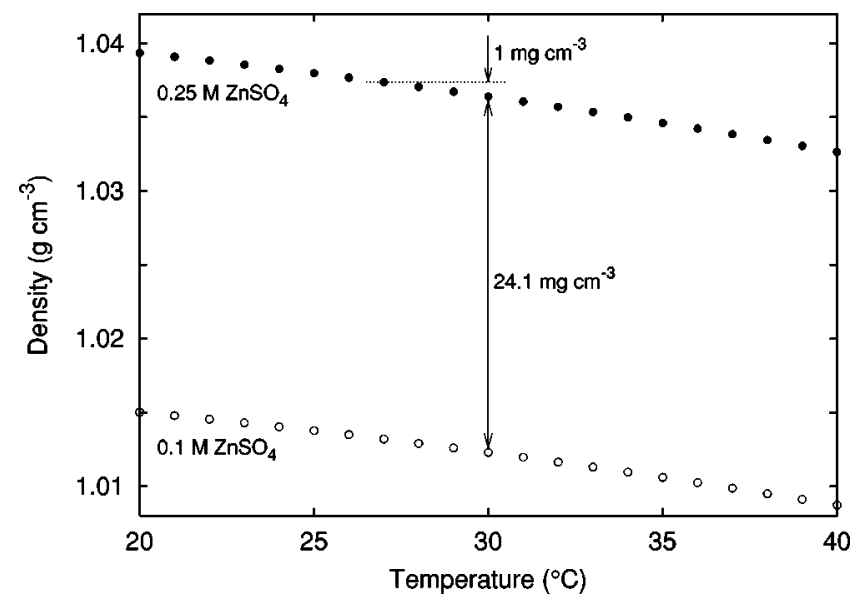

FIG. 8. Temperature dependency of the densities at the anode and in the bulk. The correspond to $c=0.25 \mathrm{M} \mathrm{ZnSO}_{4}$, the $\bigcirc$ to $c_{\text {bulk }}=0.1 \mathrm{M} \mathrm{ZnSO}_{4}$.
At the cathode $c$ has reached zero at that time, $\rho$ of $\mathrm{H}_{2} \mathrm{O}$ is $995.68 \mathrm{mg} / \mathrm{cm}^{3}$ for $T=30{ }^{\circ} \mathrm{C}$ and $995.38 \mathrm{mg} / \mathrm{cm}^{3}$ for the $T=31{ }^{\circ} \mathrm{C}$ at the maximum. This results in a $2 \%$ contribution of the temperature gradient to the total density contrast.

As our applied potential is above average for standard electrodeposition experiments, we conclude that temperature inhomogeneities will only weakly contribute to the density driven convection rolls. This result justifies with hindsight the use of the theoretical description of Chazalviel et al. [18]. Finally it should be remarked that morphologies such as stringy [30], where the zone of active growth is restricted to few small spots with very high local current densities, may differ substantially from our results.

\section{ACKNOWLEDGMENTS}

We want to thank Jürgen Fiebig from InfraTec for his friendly support. We are also grateful to Niels Hoppe and Gerrit Schönfelder from IMOS, Universität Magdeburg for their assistance with the density measurements. This work was supported by the Deutsche Forschungsgemeinschaft under Project No. FOR 301/2-1. Cooperation was facilitated by the TMR Research Network FMRX-CT96-0085: Patterns, Noise \& Chaos.

\section{APPENDIX}

The standard enthalpy of formation $\Delta_{\mathrm{b}} H^{\ominus}$ of $\mathrm{Zn}^{2+}$ ions in an infinitely diluted solutions is $-153.89 \mathrm{~kJ} / \mathrm{mol}$ [31]. It contains three different contributions: (1) the energy necessary to liberate an atom from the surrounding lattice, (2) the energy needed to ionize the atom, and (3) the hydration energy, which is set free when the water dipoles surround the ion. Only the last term depends on the concentration of the solution, decreasing with the number of ions already dissolved.

As the reaction rate at the electrodes is directly proportional to the electrical current $I$, so is the chemical power $\dot{Q}_{\text {chem }}$ :

$$
\dot{Q}_{\text {chem }}=\frac{\Delta_{\mathrm{b}} H^{\ominus}}{F z} I \text {, }
$$

where $F$ is the Faraday constant $(96485 \mathrm{As} / \mathrm{mol})$ and $z$ the charge number. For zinc we derive $\dot{Q}_{\text {chem }} / I$ $=-0.8 \mathrm{~mW} / \mathrm{mA}$. In the vicinity of the anode this number leads to additional heating due to the transformation of chemical energy. At the cathode the chemical energy stored in the newly produced zinc has to be subtracted from the overall heat production, but does not lead to a direct cooling of the cathode.

With our experimental conditions an average current of 30 $\mathrm{mA}$ feeds an electrical power of $600 \mathrm{~mW}$ into the cell. Compared to that $\dot{Q}_{\text {chem }}$ is $24 \mathrm{~mW}$ which is about $4 \%$ of the electrical power.

At the cathode the ion concentration drops fast to zero, which justifies the approximation of an infinitely diluted solution. At the anode the ion concentration increases during the whole run of the experiment. Therefore the hydration 
energy of the newly produced $\mathrm{Zn}^{2+}$ ions decreases and in consequence the heat, which is released from the chemical reaction decrease as well. So the overall heat production from chemical energy at the anode is smaller than estimated here and therefore mostly irrelevant for the energy balance. This conclusion agrees with the fact that the anode is the coldest point of the cell as clearly shown in Fig. 3, in spite of this extra heat production there.
[1] F. Argoul, A. Arneodo, G. Grasseau, and H. L. Swinney, Phys. Rev. Lett. 61, 2558 (1988).

[2] A. Kuhn and F. Argoul, J. Electroanal. Chem. 397, 93 (1995).

[3] V. Fleury, M. Rosso, and J.-N. Chazalviel, Phys. Rev. A 43, 6908 (1991).

[4] A. Kuhn and F. Argoul, Phys. Rev. E 49, 4298 (1994).

[5] M.-Q. López-Salvans, F. Sagués, J. Claret, and J. Bassas, Phys. Rev. E 56, 6869 (1997).

[6] D. Barkey, F. Oberholtzer, and Q. Wu, Phys. Rev. Lett. 75, 2980 (1995).

[7] F. Argoul, E. Freysz, A. Kuhn, C. Léger, and L. Potin, Phys. Rev. E 53, 1777 (1996).

[8] J. Jorne, Y.-J. Lii, and K. E. Yee, J. Electrochem. Soc. 134, 1399 (1987).

[9] L. López-Tomàs, J. Claret, and F. Sagués, Phys. Rev. Lett. 71, 4373 (1993).

[10] D. P. Barkey, D. Watt, and S. Raber, J. Electrochem. Soc. 141, 1206 (1994).

[11] M.-Q. López-Salvans, P. P. Trigueros, S. Vallmitjana, J. Claret, and F. Sagués, Phys. Rev. Lett. 76, 4062 (1996).

[12] M. Rosso, E. Chassaing, and J.-N. Chazalviel, Phys. Rev. E 59, 3135 (1999).

[13] M. Schröter, K. Kassner, I. Rehberg, J. Claret, and F. Sagués, Phys. Rev. E 65, 041607 (2002).

[14] V. Fleury, J.-N. Chazalviel, and M. Rosso, Phys. Rev. Lett. 68, 2492 (1992).

[15] J. M. Huth, H. L. Swinney, W. D. McCormick, A. Kuhn, and F. Argoul, Phys. Rev. E 51, 3444 (1995).
[16] M. Rosso, J. N. Chazalviel, V. Fleury, and E. Chassaing, Electrochim. Acta 39, 507 (1994).

[17] K. A. Linehan and J. R. de Bruyn, Can. J. Phys. 73, 177 (1995).

[18] J. N. Chazalviel, M. Rosso, E. Chassaing, and V. Fleury, J. Electroanal. Chem. 407, 61 (1996).

[19] S. Dengra, G. Marshall, and F. Molina, J. Phys. Soc. Jpn. 69, 963 (2000).

[20] E. Bodenschatz, W. Pesch, and G. Ahlers, Annu. Rev. Fluid Mech. 32, 709 (2000).

[21] D. E. Cormack, L. G. Leal, and J. Imberger, J. Fluid Mech. 65, 209 (1974).

[22] J. Imberger, J. Fluid Mech. 65, 247 (1974).

[23] J. Patterson and J. Imberger, J. Fluid Mech. 100, 65 (1980).

[24] B. Boehrer, Int. J. Heat Mass Transf. 40, 4105 (1997).

[25] R. Delgado-Buscalioni, Phys. Rev. E 64, 016303 (2001).

[26] C. Léger, J. Elezgaray, and F. Argoul, J. Electroanal. Chem. 486, 204 (2000).

[27] A. M. James and M. P. Lord, Macmillan's Chemical and Physical Data (Macmillan, London, 1992).

[28] H. Lindner, Physik für Ingenieure (F. Vieweg, Braunschweig, 1989).

[29] F. Sagués, M. Q. López-Salvans, and J. Claret, Phys. Rep. 337, 97 (2000).

[30] P. P. Trigueros, J. Claret, F. Mas, and F. Sagués, J. Electroanal. Chem. 328, 165 (1992).

[31] P. W. Atkins, Physikalische Chemie (VCH Verlagsgesellschaft, Weinheim, 1990). 